\title{
Reflets
}

Revue d'intervention sociale et communautaire

\section{La formation pratique : forger des alliances entre milieux universitaires et communautaires}

\section{Dominique Mercure, Marie-Luce Garceau, Madeleine Dubois et Jacynthe}

Mayer

Volume 20, numéro 1, printemps 2014

La formation pratique : allier milieux de pratique et milieux

d'enseignement

URI : https://id.erudit.org/iderudit/1025792ar

DOI : https://doi.org/10.7202/1025792ar

Aller au sommaire du numéro

Éditeur(s)

Reflets, Revue d'intervention sociale et communautaire

ISSN

1203-4576 (imprimé)

1712-8498 (numérique)

Découvrir la revue

Citer ce document

Mercure, D., Garceau, M.-L., Dubois, M. \& Mayer, J. (2014). La formation pratique : forger des alliances entre milieux universitaires et communautaires.

Reflets, 20(1), 10-16. https://doi.org/10.7202/1025792ar 


\title{
La formation pratique : forger des alliances entre milieux universitaires et communautaires
}

\author{
Dominique Mercure et Marie-Luce Garceau, \\ École de service social, Université Laurentienne
}

Madeleine Dubois et Jacynthe Mayer, École de service social, Université d'Ottawa

La formation pratique est une assise déterminante de la formation professionnelle. Les étudiantes et les étudiants peuvent ainsi à la fois appliquer les compétences travaillées en formation universitaire et effectuer une mobilisation et une combinaison de ressources acquises en contexte réel (Le Boterf, 2002). Les diverses activités académiques liées à la pratique professionnelle sont un contexte privilégié où l'intégration théorie-pratique est possible. Quant aux stages, ils visent l'intégration dans un milieu réel et l'expérimentation de l'effet des actes professionnels dans les réalités du milieu; ils permettent aussi une réflexion sur la capacité professionnelle à intervenir de manière compétente avec des personnes et des groupes, et ce, en lien avec les codes d'éthique et de déontologie.

Cet espace de formation et de socialisation professionnelles pose de grands défis et il importe que tous les acteurs concernés accompagnent les étudiantes et les étudiants dans ce processus d'apprentissage faisant le pont entre la théorie et la pratique. Cette démarche recèle d'importantes opportunités de tisser des liens entre les établissements d'enseignement et les milieux de pratique et favorise une meilleure concertation pour former de futurs professionnelles ou professionnels compétents. 
Le présent numéro de Reflets permet de mettre en commun des expériences et des réflexions entourant la formation pratique en milieu francophone minoritaire. Les contributions d'une variété d'actrices ou d'acteurs de milieux universitaires et communautaires, des domaines du service social et de la santé ont permis de soulever plusieurs questions et de dégager des pistes pour faciliter la création de ponts entre la théorie et la pratique. Les articles soulignent l'importance d'investir dans la formation des superviseures et des superviseurs, de construire de nouvelles stratégies pour augmenter l'offre de stages, de créer un encadrement spécifique pour les stages internationaux, de favoriser des manières de rallier la communauté francophone dans le parcours d'apprentissage des stagiaires, dans un souci de répondre parallèlement aux besoins du terrain tout en formant la relève, notamment en milieu rural. De plus, les articles de ce numéro invitent à réfléchir à des préoccupations émergentes eu égard à la formation pratique :l'interdisciplinaire, l'enseignement de l'approche autochtone et l'intégration de connaissances pratiques dans un cours recherche. Par ailleurs, les témoignages d'une superviseure et d'une étudiante offrent une réflexion individuelle sur l'expérience vécue de ces rôles assumés en tant qu'accompagnante et apprentie. Plusieurs pistes de pratiques novatrices visant à relever les nombreux défis auxquels se confronte la formation pratique sont aptes à susciter des réflexions permettant d'apporter un regard renouvelé sur les besoins d'apprentissage de futurs professionnelles ou professionnels.

À titre d'entrée en matière, les propos relatés dans l'Entrevue avec madame Cécile Coderre offrent d'emblée plusieurs illustrations de sujets essentiels se situant au cœur de l'intégration entre la théorie et la pratique. Dans le cadre de ses fonctions successives de professeure à l'Université d'Ottawa, de directrice de l'École de service social et de vice-doyenne de la Faculté des sciences sociales, madame Coderre a cherché à élargir l'espace de formation et d'intégration des connaissances en mettant en place avec ses collègues une panoplie d'activités d'apprentissage favorisant la création de liens entre la théorie et la pratique et contribuant à l'approfondissement des connaissances. L'intégration entre la théorie et la pratique se construit également sur des assises de collaboration entre 
l'établissement de formation universitaire et sa communauté. Dans cette perspective, l'entrevue offre plusieurs exemples éloquents de types de collaborations avec divers milieux et expose les conditions faisant en sorte que celles-ci contribuent à personnaliser et à enrichir l'expérience d'apprentissage des étudiantes et des étudiants tout en étant bénéfiques pour la communauté.

Rédigé par Dominique Cardinal, Lucie Couturier, Jacinthe Savard, Manon Tremblay et Mélanie Desmarais, l'article du Dossier sur la supervision des stagiaires souligne comment celleci permet l'intégration des savoirs essentiels à la pratique et au développement d'une identité professionnelle, d'où la primauté de son rôle dans le développement de compétences et le maintien de normes professionnelles. Les auteures traitent de l'importance d'acquérir des habiletés propres à la supervision et notent que des lacunes sur ce plan peuvent entraîner des répercussions sur l'apprentissage. Afin de soutenir la formation en supervision, le Consortium national de formation en santé (CNFS) — volet Université d'Ottawa, a développé un programme intitulé L'art de superviser des stagiaires. Fondé sur des principes andragogiques, celui-ci s'appuie sur l'apprentissage expérientiel et sur un modèle de développement de compétences en supervision et il est offert en présentiel et en ligne aux professionnelles et aux professionnels de la santé et des services sociaux des communautés francophones minoritaires au Canada. Les auteures présentent l'évaluation faite de ce programme. Les résultats montrent que la formation est de belle facture et qu'elle répond aux besoins des francophones en matière de supervision, et cela d'autant plus lorsque les ressources pédagogiques et financières dans une région ou un milieu sont inadéquates. Malgré certains désavantages et défis posés par ce type de formation en ligne, le niveau de satisfaction demeure élevé. Quant aux apprentissages issus de la formation, ils sont nombreux et permettent notamment aux personnes qui supervisent d'offrir une meilleure rétroaction, de bien clarifier les attentes et d'effectuer de meilleures évaluations du rendement, établissant ainsi une meilleure relation avec leurs stagiaires. Finalement, les auteures indiquent que les formations de base ou avancées qui sont offertes par le CNFS permettent de pallier les 
besoins des personnes francophones qui supervisent des stagiaires à la grandeur du pays.

L'article signé Hélène Albert, Isabel Lanteigne et Lise Savoie présente les dilemmes éthiques rencontrés en contexte de stages internationaux. Les auteures abordent les enjeux qui y sont rencontrés par les stagiaires en travail social et la nécessité d'une préparation adéquate permettant d'y faire face. Comme cadre d'analyse facilitant la réflexion sur les pistes de résolution des dilemmes rencontrés, elles proposent l'intersectionnalité, une approche qui tient compte des identités multiples et des structures de domination. Plusieurs situations concrètes faisant partie des défis auxquels sont confrontés les stagiaires à l'international sont présentées. Pour répondre aux dilemmes soulevés par ces défis, les auteures ont développé un modèle de délibération éthique permettant aux stagiaires de faire une analyse structurée de la situation vécue. Elles mettent en évidence les différentes dimensions sur lesquelles les stagiaires devraient s'appuyer afin de résoudre les dilemmes, notamment les personnes impliquées, les émotions, les normes professionnelles, sociales, juridiques et culturelles, les valeurs personnelles, professionnelles et sociales ainsi que les conséquences positives et négatives. En somme, les auteures suggèrent que cette intégration de l'analyse intersectionnelle à la délibération éthique permet d'éviter des pratiques oppressives.

À ces articles du Dossier s'ajoutent ceux de la rubrique Des pratiques à notre image, plus nombreux dans ce numéro. En effet, le succès de la formation pratique faisant partie d'un programme d'études universitaires repose sur un travail assidu de collaboration impliquant une variété d'actrices et d'acteurs, qu'ils soient membres du corps professoral ou de l'administration de l'université ou professionnelles et professionnels œuvrant à divers titres au sein d'une variété d'organismes, d'établissements et de réseaux communautaires. Il nous apparaissait donc essentiel qu'un numéro sur la formation pratique présente le point de vue et les expériences liées à la formation pratique d'un éventail de ces collaboratrices et collaborateurs fondamentaux travaillant au sein d'une variété d'organismes au Canada, ainsi que le témoignage et les réflexions de stagiaires. 
L'article d'Hélène Archambault, Janelle de Rocquigny, Halimatou Ba, Florette Giasson et Gisèle Lapointe présente un projet-pilote en santé communautaire de l'Université de SaintBoniface. Il a pour but de préparer les étudiantes et les étudiants de différentes disciplines à travailler en équipe transdisciplinaire. Ce projet fait partie d'un cours universitaire intégrant la théorie et la pratique, dans lequel les étudiantes et les étudiants sont jumelés à des projets communautaires touchant les populations vulnérables ou en situation minoritaire au Manitoba. Les auteures mettent en évidence les enjeux importants associés à l'implantation d'un tel projet. Les retombées tant auprès des organismes partenaires que de la population étudiante permettent de considérer ce projet comme prometteur pour la formation pratique des étudiantes et des étudiants.

L'initiative d'intégration d'une composante d'expérience communautaire à la formation médicale est aussi fort intéressante et pertinente. Comme le soulèvent Jacinthe Beauchamp, Monique Daigle et Micheline Boucher du Centre de formation médicale du Nouveau-Brunswick, cette immersion hors des murs académiques aura permis aux étudiantes et aux étudiants d'être exposés à diverses communautés et d'en comprendre les particularités, autant celles inhérentes à un milieu de pratique que celles liées au milieu de vie. Cette initiative intégrée dans leur programme et couplée à des activités de bénévolat leur aura permis de rencontrer des patients hors du milieu clinique et ainsi de mieux comprendre les réalités de leur environnement, dont plus particulièrement celles du milieu rural et les défis de santé qui y sont associés. L'apprentissage associé aux services communautaires comporte des défis d'organisation, mais il n'en demeure pas moins prometteur.

L'article de Lynn Casimiro, Geneviève Grenier, Manon Tremblay, Mélanie Desmarais et Denise Beaulieu présente quant à lui une nouvelle approche visant à augmenter la capacité d'accueil de stagiaires tout en contrant les défis de recrutement dans les stages en milieux francophones. Combinant des activités de supervision individuelle et de groupe, cette approche est novatrice dans le cadre de stages en nutrition offerts en milieu hospitalier (Hôpital Montfort) du Programme de formation spécialisé en sciences de 
la nutrition de l'Université d'Ottawa. L'article rend compte des points forts et des limites de ce projet pilote, et fait notamment l'observation que la présence du groupe a été une source de soutien et un lieu propice à de nouveaux apprentissages. Par contre, les difficultés à composer avec les attentes diverses et nombreuses de la part des différents superviseures ou superviseurs sont contrées par la valeur ajoutée du rôle de préceptrice-coordonnatrice ou de précepteur-coordonnateur. Ainsi, l'expérience démontre que ce type de supervision dépasse la simple réponse à la pénurie de places de stages et de personnel qualifié apte à superviser pour innover dans la manière d'offrir les stages.

Katharine Larose-Hébert présente une démarche pédagogique ayant été utilisée dans le cadre d'un cours de méthodologie de recherche en service social, au premier cycle universitaire à l'Université d'Ottawa. Les étudiantes et les étudiants de ce cours ont mené une recherche qualitative abordant le sujet de la conciliation des savoirs pratique et théorique au sein d'un programme en alternance en service social. Plusieurs compétences ont été développées au cours de cet exercice pratique. Il s'agit d'une initiative originale qui fut d'ailleurs fortement appréciée des étudiantes et des étudiants.

Rares sont les travaux en français qui discutent de la formation pratique au sein d'un programme universitaire de service social autochtone. Les professeures Taima Moeke-Pickering et Cheryle Partridge du School of Native Human Services de l'Université Laurentienne exposent comment la vision du monde et les enseignements autochtones sont intégrés dans la formation pratique faisant partie du baccalauréat spécialisé de leur école. Pour ce faire, les auteures font un bref détour sur l'historique et la raison d'être du programme. Elles insistent sur l'importance de l'enseignement traditionnel, les préceptes issus de la Roue de médecine et les Enseignements des sept grands-pères dans l'ensemble du programme comme encadrement du passage de la théorie à la pratique. Elles décrivent également l'incorporation et la mise en pratique des préceptes de la Roue de médecine, incluant le Rituel de purification, et les Enseignements des sept grandspères dans la préparation à la formation pratique et dans le stage. 
L'article de Chantal Joly présente le point de vue d'une superviseure au sujet de quelques-uns des enjeux inhérents à l'accueil et à l'intégration d'une stagiaire au sein d'un contexte organisationnel comportant, comme tout système, une culture particulière, avec des défis qui lui sont propres. En apportant un éclairage sur la nature et la portée des questions dont doit se soucier toute personne appelée à superviser des stagiaires, l'article de madame Joly contribue à favoriser une meilleure compréhension des soutiens nécessaires, à la fois au sein des contextes de travail et de la part des responsables universitaires, pour assurer la réussite d'un programme de formation pratique.

Les stages de formation offrent aux étudiantes et aux étudiants un contexte de socialisation professionnelle au sein duquel ils sont appelés, notamment, à se confronter aux réalités et aux complexités du terrain, à se mesurer aux défis d'adaptation et d'applicabilité de leurs savoirs théoriques, mais également à faire face à des situations qui ébranlent parfois leurs valeurs et leur vision du monde. Comme le relate Mélanie Parent dans l'article décrivant son expérience comme stagiaire, ces défis prennent une signification d'autant plus importante lorsque le stage s'effectue dans un pays éloigné, avec une culture et des coutumes différentes de celles qui sont familières.

Les personnes s'intéressant à la formation pratique et aux questions d'intégration théorie-pratique déplorent souvent la pénurie d'écrits et de recherches dans ce domaine fort important de l'éducation à la pratique professionnelle, plus particulièrement dans des contextes spécifiques, s'adressant à des groupes minoritaires, des milieux ruraux ou des territoires géographiquement dispersés. Les contributions de ce numéro cherchent à faire avancer la réflexion sur ces questions. Nous remercions leurs auteures d'avoir contribué à la diffusion de nouvelles connaissances et de nouvelles pratiques dans le domaine de la formation pratique.

\section{Bibliographie}

LE BOTERF, Guy (2002). Développer la compétence des professionnels, Paris, Éditions d'Organisation, $311 \mathrm{p}$. 